\title{
Augmented reality meeting table: a novel multi-user interface for architectural design
}

\author{
A. Penn, C. Mottram, A. Fatah gen. Schieck, \\ M. Wittkämper ${ }^{1}$,M. Störring ${ }^{2}$, O. Romell ${ }^{3}$, A. Strothmann ${ }^{4}$, F. Aish ${ }^{5}$,
}

The Bartlett Graduate School, University College London,UK

${ }^{1}$ Fraunhofer FIT, ${ }^{2}$ Aalborg University, ${ }^{3}$ SaabAvionics, ${ }^{4}$ Linie4 Architekten, ${ }^{5}$ Foster and Partners

Key words: Design Collaboration, Tangible Interface, Gesture, Agent Simulation, Augmented Reality.

\begin{abstract}
Immersive virtual environments have received widespread attention as providing possible replacements for the media and systems that designers traditionally use, as well as, more generally, in providing support for collaborative work. Relatively little attention has been given to date however to the problem of how to merge immersive virtual environments into real world work settings, and so to add to the media at the disposal of the designer and the design team, rather than to replace it. In this paper we report on a research project in which optical see-through augmented reality displays have been developed together with prototype decision support software for architectural and urban design. We suggest that a critical characteristic of multi user augmented reality is its ability to generate visualisations from a first person perspective in which the scale of rendition of the design model follows many of the conventions that designers are used to. Different scales of model appear to allow designers to focus on different aspects of the design under consideration. Augmenting the scene with simulations of pedestrian movement appears to assist both in scale recognition, and in moving from a first person to a third person understanding of the design. This research project is funded by the European Commission IST program (IST-2000-28559).
\end{abstract}




\section{INTRODUCTION}

\subsection{The co-evolution of design theory and computing}

The way in which designers design has been the subject of considerable investigation and debate over the last 50 years. Much of this debate has been closely associated with the development of design computing which by its nature has required us to understand and define exactly what we mean by design. In this paper we report on the ARTHUR project, the latest in a long line of research projects aimed at developing computing for design, which perhaps helps throw further light on the nature of design itself.

In the early years of architectural research, the contention was that formalised procedures or 'design methods' would bring rationality to the design process. In this view the methods of operational research, developed with such success to provide logistic support for the military during the war, could be brought to bear on design which until then had been largely seen as an intuitive (and therefore unscientific) activity (Rittel, Webber, 1973). Herbert Simon championed the cause during the 1950s (Simon, 1969) and Christopher Alexander during the 1960s, and by the time of the Oxford Conference on Design Methods in 1963 this view was well established. Although it was noted that design methods might inhibit imagination and creativity, it was felt that by integrating brainstorming into the process even this problem could be overcome (Jones, 1963). However, only a decade later this view had come to be comprehensively challenged from a number of directions.

Alan Colquhoun noted the centrality of typology and precedent in design thinking (Colquhoun, 1967). As often as not design proceeded by reference to past 'good solutions' to a problem, or through an analysis of the range of solution types that might be applicable to a specific problem. Meanwhile Bill Hillier developed a critique of design method based on Popper's analysis of scientific method (Hillier, Musgrove, et al., 1972; Hillier, Leaman, 1974). He proposed that knowledge of the effects of local design moves on global outcomes was the specifically architectural knowledge that was needed in design. By the 1980s even those who had been the originators of the Design Methods movement had completely reversed their positions: "in the seventies I reacted against design methods. I dislike the machine language, the behaviourism, the continual attempt to fix the whole of life into a logical framework." (Jones, 1977); "Jacobson: In what areas should future work centre in design methodology? Alexander: I think I should be consistent here. I would say forget it, forget the whole thing." (Alexander, 1971). Analysis of design processes evolved radically during this period through a recognition of the inadequacy of the linear process characterised in the early 
days of Design Methods (eg. Archer, 1979) and the realisation that design processes were both fuzzy and iterative in nature (Checkland, 1981; Checkland, Scholes, 1990). In this analysis it was clear that the path followed by the design process was not from a well defined problem to an optimal solution, but as often as not the reverse: through an exploration of possible solutions to a better definition of the brief or design problem at hand (Rittel, Webber, 1973). Herbert Simon noted that architectural design was characterised, like chess, by problems that are "well structured in the small but ill structured in the large" (Simon, 1973). That is, whilst in detail it is often possible to give a well constrained definition of the design problem at hand, it is often impossible to do so for a whole design.

Attention had been drawn to the important distinction between design as a procedure and as a cognitive process by Hillier at UCL (Hillier, Musgrove, et al., 1972), but an understanding of the latter was developed through the analysis of design activity by Donald Schoen and his colleagues at MIT. Their key contribution was to formalise from a theoretical standpoint the notion of 'knowledge in action'. This is that class of knowledge that is captured not in words, but in actions or 'know-how'. For Schoen, design, and especially the interaction between the designer and the plastic media with which they work during design - sketches and maquettes - is largely learned and practiced through action and reflection. The sketch is a concretisation of an abstract idea of a building, which can then be reflected on, and it is this dialogue which characterises an important dimension of the design process (Schoen, 1983).

Schoen's analysis goes further, however, to discuss the education of the 'reflective practitioner'. He notes that knowledge in action must be developed through action itself, and he proposes that the design studio and the design jury are typical means to accomplish this. It is by presenting a design to colleagues and tutors that the implicit understanding of what design entails is built up (Schoen, 1987). This sets out design education and practice (along with most other forms of professional practice) as a primarily social and collaborative activity. The work of Tom Allen on innovation in engineering design is relevant in this context (Allen, 1984). Allen's studies of large defence engineering projects found that despite the hopes of the brainstorming enthusiasts, innovative ideas in design projects often arose through the import of ideas from outside the group. Innovation, it seems, is substantially a product of collaboration, and the most productive collaborations involve individuals who bring radically different mindsets to the problem (Penn, Desyllas, et al., 1999).

Others have noted that design practice is characterised by a dichotomy around the design-meeting table, between the 'form generators' and the 
'analyst critics' (Hillier, 1998). In this situation design proposals (generated by the 'designer') are reviewed by a range of experts: engineers, cost consultants, contractors and client or user representatives. Here the key capacity of the designer is to listen to the critique, to internalise its consequences and to use this knowledge to help refine the problem definition and to narrow the range of solution types that need to be developed. The process has been described as akin to solving Rubik's cube. Since each domain that must be resolved is independent, but all interact through the proposed built form, as a move is made to resolve one issue it creates problems for another domain. It is for this reason that design requires both a broad range of expertise around the table and an iterative process (Penn, Treleavan, et al., 1996). However, it is also clear that collaboration in design is itself a reflective activity in Schoen's terms. The process of listening to domain experts and internalising the dynamics of different form/function relationships is itself learned through action and largely an implicit skill. Habraken and Gross investigated these collaborative skills through game play (Habraken, Gross, 1988), and found that experience of collaboration in games improved the effectiveness of team based design work. In a more recent analysis of historical design projects, Chengzhi Peng reviewed the importance of different representational media in design collaboration, including the use of overlays, scores and shared modelling spaces (Peng, 1994).

By the 1990s the true complexity of architectural design processes was well established and the research field had divided to address the range of specific sub themes that seemed to form part of the whole. The field was characterised, it seemed, by a plethora of dichotomies: should research focus on the process or the product? Was design bottom up or top down? Intuitive or rational? Art or science? Individually or socially constructed? Emergent or rule governed? Which comes first, the problem or the solution?

Answers to these binary oppositions were generally 'both', perhaps presaged most eloquently by Robert Venturi. Architectural design, it seemed, was consistent in its need to span and work from both ends of any axis, and creativity thrived on the contradictions involved in this (Venturi, 1966).

Among the key components of the research field was the investigation of part-whole relationships both in analytic mode (eg. Space syntax studies Hillier, Hanson, 1984; Hillier, 1996) and in generative mode (eg. Shape grammars - Stiney, March, 1981). Both of these approaches (and others akin to them) lent themselves to computation, and in fact required computation for their execution. At the same time, in architectural practice, the development of computing to assist in the organisation and management of production information had taken over the way that this aspect of the process 
was organised. The effect of Computer Aided Design (CAD) in the office was to bring into clear focus the distinction between drawing (or sketching) as a cognitive activity supporting design, and drawing as method of communication of production information (Lawson, 1990). The latter is now almost entirely computer based in the architectural profession, but the former remains firmly rooted in the media of the plastic arts (including of course, digital media).

Developments in computing for architectural design since the 90s have tended to follow one of a number of main lines: three dimensional modelling for visualisation; parametric and scripted modelling; analysis and simulation of functional performance for concurrent engineering; case based or precedent databases; and, evolutionary or optimised form generation. Of these, one of the great hopes of the 90 s was that improvements in visualisation, and Virtual Reality (VR) in particular would help the 'intuitive' aspect of the creative process. By allowing designers to inhabit and visualise their emerging design, now possible at 1:1 scale through fully immersive VR, it was hoped that computing could be brought to the service of design rather than just production information.

These hopes for fully immersive VR in architectural and engineering design have not yet been borne out in reality for a number of reasons, including cost and the unfeasibility of working in a completely immersive environment. Full immersion has therefore remained of greater academic than practical interest. More recently, the focus of research has shifted again, towards lower cost systems and the merging or augmentation of the real work setting with virtual media.

Amongst recently developed technologies, Augmented Reality (AR) offers a number of attractive properties which may help support design processes as we are coming to understand them. AR as a field has developed rapidly over the past ten years (Azuma, 1997). AR systems and technologies originated in the head-up display technologies developed for defence (Wanstall, 1989) and were introduced into a broader engineering context in 1992, when they were developed to support industrial manufacturing processes by Boeing (Caudell, Mizell, 1992). Since 1996 AR and its applications have been investigated for various surgical applications such as needle biopsy and minimally invasive treatment, where, by combining Computer Tomography, Magnetic Resonance and Ultrasound Imaging, with the real scene, doctors are provided with information superimposed on the patient (Weghorst, 1997; Fuchs, Livingston, et al., 1998).

In the architectural and construction field it has been suggested that an AR system might give users "X-ray vision" inside a building, allowing them to see where the pipes, electric ducting, and structural supports are inside 
walls and above ceilings (Feiner, Webster, et al., 1995; Webster, Feiner, et al., 1996) or generate 3D models of pipelines in a factory and register them with the user's view (Navab, Bascle, et al., 1999). In all of these applications the main thrust of the research is to use AR techniques to augment physical objects in the environment with additional information or views of their internal, hidden structure or anatomy. The feasibility of optical see-through (as opposed to video see-through) AR for these applications is severely limited by current wide area head tracking technologies needed if the virtual and models are to be properly registered with respect to the real environment (Azuma, Baillot, et al., 2001). To date these applications have remained laboratory based, however the development of computer vision systems for head tracking using head mounted cameras have been shown to allow viable frame rates outside the laboratory (Argyros, Trahanias, et al., 1998).

A number of current and recent research projects are directly relevant to the augmented reality approach presented in this paper:

BUILD-IT (Rauterberg, Fjeld, et al., 1997) a multi-user planning tool using a 2D projection on a table top. Additionally, a video camera is used to track manipulations of a small, specialized brick that can be used as a "universal interaction handler". A second, vertical projection screen behind the table provides a 3D view of the virtual scene.

MagicMeeting (Regenbrecht, Wagner, et al., 2002) supports product review meetings by augmenting a real meeting location. Virtual 3D models are loaded into the environment from desktop applications or Personal Digital Assistants (PDAs). The MagicMeeting system explores several interaction techniques, including the MagicBook metaphor (Billinghurst, Campbell, et al., 2000), annotations and a clipping plane tool. The 3D models are linked to real placeholder objects to create a tangible interface (Ishii, Ulmer, 1997).

MARE (Grasset, Gascuel, 2002) is a multi-user augmented reality system designed to be used in any application domain. The table space is divided into two parts: a personal area for the private real objects (pen, PDA, cup) and virtual ones (private menus); and a shared interactive space.

MIXDesign (Dias, Santos, et al., 2002) provides a Mixed-Reality system oriented towards tasks in Architectural Design. The system explores tangible interfaces using AR Toolkit patterns on a paddle and gestures.

TILES (Poupyrev, Tan, et al., 2000) is a collaborative tangible AR that allows two-handed interactions. The interaction techniques are aimed at the application for rapid prototyping and evaluation of aircraft instrument panels. The spatial interaction is in $3 \mathrm{D}$ allowing the user to manipulate virtual data on any working surface.

URP (Underkoffler, Ishii, 1999) is an application for urban planning and design. The infrastructure allows physical architectural models placed on a 
architectural design

projected table surface to cast shadows accurate for any time of day; to throw reflections off glass facade surfaces; to visualise a simple 2D Computational Fluid Dynamics (CFD) analysis of wind flow. This is accomplished using $I / O$ Bulb techniques to attach projected forms to physical architectural models.

In these projects four main concerns are notable:

- the use of tangible interfaces to engage both hand and eye in response to the cognitive importance of action in design;

- the merging of environments with visualisations of functional performance;

- a catholic approach to mixing of different conventional, digital, VR and AR media;

- the support of collaboration through sharing of a single representation on a 'table-top' or large screen visualisation.

This is the context within which the ARTHUR project described in this paper is set. By using optical see through Augmented Reality (AR) displays the ARTHUR system aims to put virtual 3D models on the designer's meeting table alongside conventional media. In the next section we describe the ARTHUR system in detail. We then review early findings from the system in use, including qualitative feedback from our first user tests, before finally drawing conclusions about the light these new media throw on our understanding of the nature of the design process.

\section{ARTHUR}

Imagine the scene. A design meeting consists of a group of people seated around the meeting table. On the table are various drawings, a polystyrene sketch model, photographs, perhaps some material samples, and inevitably a roll of layout paper and some felt tip pens. As people discuss the scheme they use the drawings and model to illustrate their point. From time to time someone will sketch something - a possible solution to a problem. Often they will do this alone as the conversation proceeds before tabling it to help others understand their proposition. Sometimes the sketching is done in front of the group where the act of drawing is itself an aid to communication. The poly model becomes the focus of attention, a block is moved and another cut to change its shape. One of the consultants around the table raises a point and illustrates it with reference to a spreadsheet calculation on her laptop. However, there is a key concern. Something that can only be resolved through a simulation based on the new plan. Tasks are allotted and decisions postponed until this can be done. 
Now imagine that everyone has lightweight clear glasses; these have a pencil camera on each side and are connected to a computer. The glasses are augmented reality displays - the kind that the military use to give head-up display information to fighter pilots - the cameras track the scene and allow a 3D computer model generated from the CAD system to be kept still on the table as the user moves around it. The cameras also recognise placeholders on the table and the user's hand gestures. These are used to interact with the model. Moving a placeholder or making a gesture changes the model. As the model is changed there is an instantaneous link to the consultant's spreadsheet and its results are recalculated. The results of analysis and simulation can be rendered into the model on the table, and so long as calculation time allows, these can be updated as the model is manipulated. This is the ARTHUR concept (Broll, Störring, et al., 2003). It is not a replacement for traditional media, but an attempt to bring the computer - as an interactive and creative medium in its own right - onto the meeting table.

\subsection{System architecture}

The ARTHUR system consists of four main components. An optical seethrough AR Head-Mounted Display (HMD) prototype has been developed by Saab Avionics specifically for the project. This incorporates head mounted stereo cameras to provide Computer Vision (CV) based object tracking of 'placeholder objects' and pointing devices for use in interaction with the virtual media, as well as finger tracking and gesture recognition. The CV system is also used to track the users head position and so to keep the virtual model stable in the real environment from the user's point of view. An integrated software framework provides for visualisation, distributed communication for multiple users and CAD integration through an Application Programming Interface (API). A graphical language (GRAIL) has been developed to allow users to configure the relationships between inputs and outputs within the system, including through external applications. Each of these components is described in detail below.

\subsection{AR display}

The ARTHUR head-mounted display is a transparent high-resolution binocular 3D HMD. The image is generated on two independent full color 1280x1024 pixels (SXGA) Liquid Crystal on Silicon micro displays. The ARTHUR HMD has excellent image quality, high brightness and contrast.

The image can be shown superimposed on the environment with up to 35 $\%$ see-through or fully immersed. The ARTHUR HMD is designed for a 46degree diagonal $100 \%$ stereo overlap field of view. A $50 \%$ overlap can also 
architectural design

be used, giving a 54-degree horizontal or 60-degree diagonal field of view. It features a patented optical design that combines a wide field of view with high transparency see-through and a patent pending head fitting system with easy adjustments, low weight and eyeglass compatibility for most users.
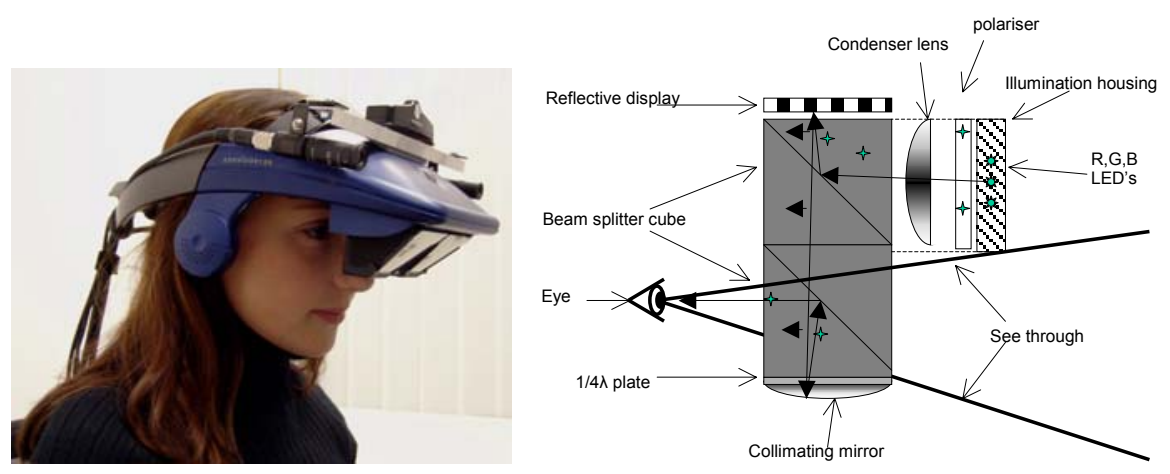

Figure 1 The optical see-through HMD with mounted cameras and tracker unit

\subsection{Computer Vision}

Computer Vision (CV) techniques take input from stereo Head-Mounted Cameras (HMCs) and a fixed camera, and are used to track the movements of real world items (placeholder objects and two hand held pointers) and to recognise hand gestures. The computer vision based approach allows users to interact without cables or sensors connected to the objects they manipulate to create an interface. The hope here is that interaction will be more natural and intuitive.

\subsubsection{User Interface Devices: Placeholder and Pointer Tracking}

The user interface devices are dedicated objects that are tracked by the computer vision system using colour and shape information. There are two types of device: placeholder objects (PHOs) and wand-like pointers.

Placeholder objects are tracked in the table plane, in two translational and one rotational degrees-of-freedom giving 3DOF information. They are of a convenient size to be grasped and moved by users. More than ten placeholders may be used concurrently. The users may take a placeholder object, associate it with any virtual object and move this virtual object by moving the placeholder object, thereby creating a direct manipulation interface. Alternatively, any one of the 3DOF can be associated with any object attribute for any virtual object. Thus, for example, a PHO's orientation could be used to set a virtual object's height or colour. 
The pointers are tracked in 5DOF - all except roll - by the head mounted cameras. This has the advantage that the pointer is always tracked when it is in the user's field of view. The pointers have three buttons for functionalities such as pick or select. Users may select and manipulate the shape of virtual objects with a pointer or use it to navigate in virtual menus.

\subsubsection{Gesture Recognition}

Two types of gestures can be used, static command gestures and 5DOF fingertip tracking. Both gesture types are tracked by the head-mounted cameras and the CV system.

A set of five static command gestures have been implemented. The number of fingers shown to a head-mounted camera is interpreted as a gesture. These command gestures can be associated with a change of mode in the user interface. For instance, they can be used to display or hide a popup menu, or to execute functions such as cut-copy-paste. The 3D position of the users index fingertip and the direction their finger is pointing is also tracked using the head mounted cameras. Moving the thumb generates a 'click' event. Fingertip tracking may be used to draw a line in space or to select items in pop-up menus, select objects and execute actions.
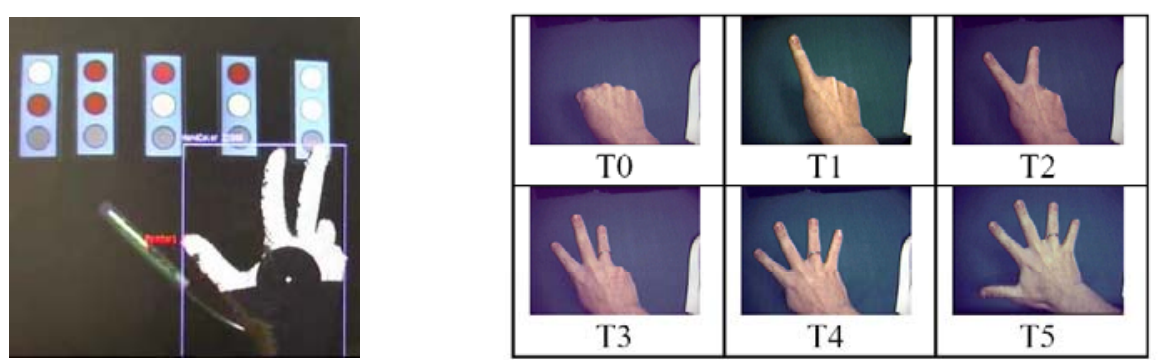

Figure 2 Left: 3 DOF PHOs and a 5 DOF pointer. Right: Static command gestures

\subsubsection{Computer Vision Based Head Tracking}

In an AR system, fast, accurate, and jitter-free tracking of the user's head pose (position and orientation) is crucial if the virtual objects in the display are to appear stable in the real environment. In ARTHUR the head pose was originally tracked using a commercial tracking system. In order to reduce jitter the head pose is now tracked using the HMCs and the two sources of information are fused. The head tracking via the HMCs is accomplished using the positions of the PHOs found using the fixed camera system. 
architectural design

\subsection{AR Framework Components}

The AR framework is a $\mathrm{C}++\mathrm{API}$, which provides the basis for bringing all the individual ARTHUR components together. It consists of three main elements:

\subsubsection{D-Visualization}

The 3D-visualisation component renders geometric objects in 3D using head mounted displays or other output devices. Rendering can be either stereoscopic (quad-buffered or dual screen) or monoscopic. Augmentation within ARTHUR is usually achieved using see-through augmentation, i.e. the virtual image is superimposed optically on the real environment. The 3Dvisualisation component however, additionally supports video augmentation, where the virtual image is superimposed on a video image from a headmounted camera. This may be used for screen-based presentations or larger projections to show people not wearing an HMD, what is currently visible to those participants. The scene contents displayed by the visualisation component are based on Virtual Reality Modelling Language (VRML) 97. In the overall system, one visualisation component exists for each individual user, allowing rendering to be performed locally. In order to achieve this, the scene graphs are replicated among the individual visualisation components and kept synchronised by distributing changes to geometry.

\subsubsection{Distribution and Communication}

The AR framework provides the distribution and communication mechanisms needed to connect the input devices such as head tracking, computer vision input (placeholder tracking, pointer and finger tracking, and gesture recognition) to other system components (e.g. the 3D stereo visualisation components). This allows the visualisation components to adapt their current viewing position and orientation to the tracking input. Additionally this distribution mechanism is also used to keep the scene graphs of multiple users synchronised (i.e. upon changes to one local scene graph, these changes are immediately distributed to all other replicated scene graphs). Synchronised clocks between all PCs involved are required in order to resolve ambiguities. The general communication mechanism used within the AR framework is Common Object Request Broker Architecture (CORBA). A universal sequencer mechanism provides an overall virtual time management for all components depending on virtual time. 


\subsection{Graphical AR Interface Language}

In ARTHUR a high level graphical interface gives non-programmer users the ability to fully configure their interactions with the system. This makes use of a Graphical Reality Augmentation Interface Language (GRAIL), which provides an interface combining both $2 \mathrm{D}$ applications and 3D manipulation and visualisation tools in the 3D space.

The GRAIL application sits on top of the AR framework and acts as a 'tool building tool' to define the properties and characteristics of the overall interaction environment. In this sense it performs as a Graphical User Interface (GUI) builder, but for AR environments and interactions.

Users can create a range of tools by graphically defining the relationship between the 3DOF PHOs and 5DOF pointers and command gestures and the virtual objects (defined as VRML nodes) or system modes. The relationships within a GRAIL configuration can easily be changed and reassigned, providing the ARTHUR system with a flexible interface that supports a rich type of interaction with the system and the virtual objects. Essentially, we provide this flexibility in an attempt to define ARTHUR as a creative or plastic 'medium' in which users are free to define their own forms of interaction between virtual and real objects and their own gestures and actions.

GRAIL also provides the user with the ability to link the ARTHUR system to external applications, such as pedestrian and environmental analysis applications, using scripting commands as an alternative to using the full API. The use of scripting commands allows these links to be made by the user at run time without the need to recompile.

\subsection{The user interface approach}

Although desktop software applications now have a well-defined set of graphical user interface concepts to work with, no common user interface paradigms have yet been defined for immersive $3 \mathrm{D}$ or AR systems.

A starting point for the design of the ARTHUR user interface was to use the architectural desktop as a metaphor, to define the characteristics of the interaction environment. The critical question however, was the degree to which an augmented reality interface to design should follow the conventions that have been developed for interaction within the architectural desktop environment, bearing in mind that the conventional architectural desktop is designed for individual interaction and the efficient management of production information rather than to support the cognitive processes of design. In view of this our approach to interface design has been through the development of a series of usage 'scenarios' in collaboration with end user 
architectural design

partners. These scenarios are then implemented using the GRAIL interface and amended in response to users feedback.

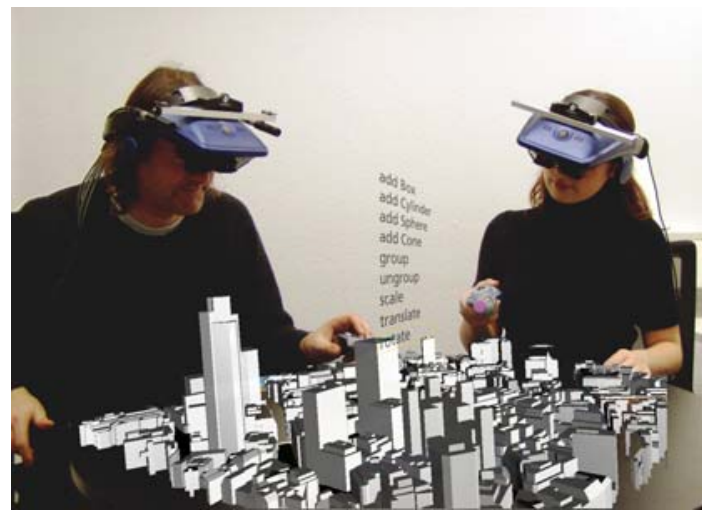

Figure 3 Collaboration within ARTHUR environment

\subsection{Designing with a live movement simulation}

Architecture differs from the plastic arts - painting and sculpture - in that in addition to its aesthetic, cultural and structural roles it is expected to perform functionally. That is, to provide shelter by modifying the environment and to support specific social functions by ordering spatial arrangement to generate or control patterns of contact between different groups of people. It is through the latter that architecture functions socially, and it is this that leads to a hospital being differently planned to an office or a prison. It has been proposed that the social function of both architecture and urban form derives from the way that the configuration of space affects the way that people are brought into contact as they move around. It is this 'construction of the interface' between different categories of people that characterises the social function of architecture at its most basic (Hillier, Hanson, 1984; Penn, Desyllas, et al., 1999).

In the development of the ARTHUR system it would clearly be impossible to develop the full range of analytic methods used even on a relatively simple building project. However it was also clear that the ability to subject a design to a simulation of functional performance was a critical component of round table collaborative design. It is for this reason that we chose to investigate the use of a pedestrian simulation to bring the ARTHUR model on the designer's meeting table to life, however, this is but one example of the kinds of analysis that could be incorporated into the system. 
In order to simulate realistic movement patterns and rates we have developed agent simulations in which an agent's forward facing field of view is sampled, and an immediate next step chosen from that field. In this we have relied on previous research that has found that visual field based movement of this sort provides a good correlate with observed aggregate movement behaviour in urban environments and buildings (Penn, Turner, 2002; Turner, Penn, 2002). In this case, however, in order to allow the environment's geometry to be varied in real time while the simulation is running, our algorithms are based on sampling the current environment rather than pre-computation of visual fields.
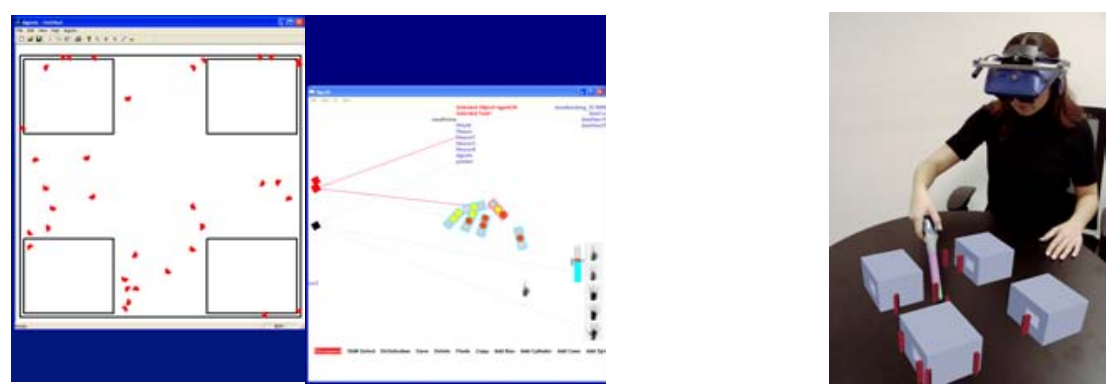

Figure 4 Left: Linking the agent software in GRAIL. Right: Adding agents using the pointer

\section{SYSTEM EVALUATION AND DISCUSSION}

\subsection{Early results of user studies}

All the main components of the ARTHUR system have now been developed and integrated, and early user evaluations of each component and of the whole system have begun. To date, three main types of user evaluation observations have been conducted. First, single user tests were conducted with subjects from our application partner organisations to evaluate usability for simple manipulations using simple geometric transforms (translation, rotation, scaling) with different interaction techniques using the pointer to navigate in virtual menus or to select and manipulate the shape of virtual objects. Here the protocol was use of the system followed by debriefing discussions. These observations must be considered only as preliminary and a part of an early prototyping development process since the subjects had been intimately involved in the specification of the system, however, they proved to be very useful in helping us to develop appropriate interaction techniques. The results of these tests were directly incorporated in the development of the system. 
The second form of evaluation was through formal user tests and associated observations and questionnaires. An experiment was designed to assess the impact of the ARTHUR environment on the level and quality of collaboration between users by evaluating the way users perform a simple task. Two teams of collaborators were first given a short period of training and time to get used to the system, and then were asked to collaborate to design a simple structure. The interaction techniques we tested were restricted to adding $3 \mathrm{D}$ objects to the common space using two types of gestures; a two-finger gesture to create an object (a simple cuboid) and a one-finger gesture to locate it. In this way the objects could be aligned and stacked to form a structure. The form of collaboration was observed and annotated by a researcher, and notes were taken of the form of interaction between the subjects, of difficulties experienced with the interface and of their comments on the system while using it. Following the session, the participants were debriefed in both a structured discussion and using a questionnaire.

The third form of user evaluation took place as a part of a public demonstration of the ARTHUR system at the Fair for Information Technology and Telecommunications (CeBit 2004) in Hanover. A large number of people participated in either a single user or in a multi-user environment depending on their preference. These users were all first time users with no previous knowledge of the system. A brief description of the system and instructions were given by a demonstrator, and then they were invited to use the system. The interaction techniques consisted of simple object creation and manipulations using simple geometric transforms (translation, rotation, scaling). Placeholder objects and pointers were used to interact with virtual menus or to select, move and manipulate the shape of virtual objects. All participants were observed, and notes were made of the difficulties they experienced and assumptions they made about the user interactions within the system. Of these subjects, ten who showed particular interest in the system were asked to provide detailed feedback in a form of a questionnaire. The main observations we report here are therefore qualitative and derived from the discussions and observations described above.

System evaluation at such an early stage in development and integration was surrounded by technical glitches and inconsistencies, and it was clear that these hampered a user's ability to evaluate the system. Having said that, we observed that with simple instructions the users were able to use gestures, pointers and placeholders effectively. All subjects found the system to be both enjoyable and to offer a potential for collaborative design. However, subjects pointed out that the use of gesture was not natural "I am used to having something in my hand while designing: a pen or a mouse". Subjects 
who used the gestures effectively were able to do this only after a period of training. This is unsurprising given the command gestures comprise a language to be learned.

Generally, it appeared difficult to understand the virtual objects as integrated in the physical environment. This might be expected since physical laws e.g. gravity and inertia, are not applied. Subjects were not always aware of the new objects that were added by another collaborator, especially when the scene was cluttered, "It was confusing to see a box suddenly appear floating".

It was difficult to understand distances, and subjects, especially those with $\mathrm{CAD}$ experience, pointed out that a common view of a situation might help collaboration "to have a view that the other user can see". This was unexpected. It appears to be partly a result of the restrictions on a user's movement in their environment imposed by the system, the weight of the HMD and its cables, and the need to keep the PHOs that sit on the table in the field of view in order to allow head tracking. Ideally, users should be able to move their heads freely and wander around the table and observe the design from different viewpoints. In addition, the appearance of the virtual objects as always superimposed on real objects, especially the user's hands, may have led to a loss of the feeling of the virtual as existing within the real world. In conclusion, users found that the properties of the interaction space, viewed through a relatively narrow field of view provided by the headset, are currently closer to those of an immersive VR than an augmented real space "I was unaware of the real environment, immersed and engaged in the task until somebody (the collaborator) talked to me". In this situation speech appeared to be crucial to subjects' perception of the social activity and to the collaboration itself.

When users are faced with simulated pedestrian movement two key factors seem to emerge. The first is that the simulation lends a sense of scale to the model through size and speed of movement of the simulation agents. The second is that it seems possible to empathise with the simulation agents, and so to 'inhabit' the virtual model at its reduced scale. Games are played by moving buildings to affect movement patterns and these games actively involve both designers and simulation agents.

\subsection{Conclusions: social organisation and play}

Every new design medium brings to light insights into the design process. The advent of CAD made it clear that the cognitive dimension of design was separate to the communication of production information, and perhaps one of the most interesting aspects of the ARTHUR development lies in what it seems to tell us about the way that designers collaborate. 
There is clearly an asymmetry around the designers' meeting table between the activities of form generation and those of critical analysis. As a system, the intention was that ARTHUR should support both aspects of design interaction. However, we have found that collaborative design is complex: form generation as an activity seems to be substantially an individual one whilst analytic criticism is a group effort. Users find it extremely hard to even think about how it might be possible to really collaborate in real time in the act of form generation itself.

At the same time, the new AR medium seems to hold out a prospect for quite different ways of working. Two different kinds of behaviour were observable in our user tests of collaboration. In the first, one member of the team would take charge of the process, and direct actions "you do this, and I'll do that". This form of organised teamwork is familiar in design teams, and is a normal feature of most architects' offices. In the second, collaborators began to play games. Here for instance they would place blocks to channel the movement of the simulated pedestrians, in much the same way that children collaborate in damming streams.

We believe that creating architectural forms and working on a task collaboratively became a game that users enjoyed and consequently this increased their level of collaboration: "it was great to see the other user and talk, and shape the design space together". Playing such games, particularly where they involve simulations of functional performance, may also promote learning. This is supported by Habraken. "the actual making of decisions about forms in space - had a strong and inevitable social dimension and as such was influenced by the way in which involved parties interacted" (Habraken, Gross, 1988). Our findings to date support this, and suggest that if technical and human factors barriers can be overcome, augmentation of the real world by digital interactive media may lead to new forms of genuinely collaborative form generation. One consequence of this is that design education may need to begin to learn some lessons from time based performance media about how to train designers to collaborate and play.

\section{Acknowledgment}

Extra contributions from I. Lindt, C. Yuan, W. Broll, T. B. Moeslund, Y.Liu, E. Granum, H. Hildmann, S. Preikschat, and H. Whitehead.

\section{REFERENCES}

Alexander, C., 1971, "The State of the Art in Design Methodology", (replies to questions by M. Jcobson), DMG Newsletter, p. 3-7.

Allen, T., 1984, Managing the Flow of Technology, MIT Press. 
Archer, L., 1979," Whatever became of Design Methodology", Design Studies,1 (1), p.17-18.

Argyros, A. A., Trahanias, P.E., and Orphanoudakis, S.C., 1998, Robust Regression for the Detection of Independent 3D Motion by a Binocular Observer, Real-Time Imaging, 4(2), p.125-141.

Azuma, R.T., 1997, “A Survey of Augmented Reality”, Presence: Teleoperators and Virtual Environments, 6(4), p. 355-385.

Azuma, R.T., Y. Baillot, R. Behringer, S. Feiner, S. Julier, and B. MacIntyre, 2001, "Recent Advances in Augmented Reality", IEEE Computer Graphics and Applications, 21 (6). P.34-47.

Billinghurst, M., S. Campbell, D. Hendrickson, W. Chinthammit, I. Poupyrev, K. Takahashi, and H. Kato, 2000, Magic Book: Exploring Transitions in Collaborative AR Interfaces, SIGGRAPH 2000 Emerging Technologies Proposal, ACM SIGGRAPH 2000.

Broll, W., M. Störring, and C. Mottram, 2003, "The Augmented Round Table - a new Interface to Urban Planning and Architectural Design”, INTERACT 2003, p.1103-1104.

Caudel, T.P., and D. Mizell, 1992, “Augmented Reality: An Application of Heads-up Display Technology to Manual Manufacturing Processes", Proceedings of the Hawaii International Conference on Systems Sciences, Maui, Hawaii, IEEE Press, p. 659-669.

Checkland, P., 1981, Systems Thinking, Systems Practice, John Wiley and Sons, Chichester.

Checkland, P., and J. Scholes, 1990, Soft Systems Methodology in Action, John Wiley and Sons, Chichester.

Colquhoun, A., 1967, “Typology and Design Method", Arena, 83, p.11-14.

Dias, J.M.S., P. Santos, and N. Diniz, 2002, "Tangible Interaction for Conceptual Architectural Design", ART 02.

Feiner, S., A. Webster, T. Krueger, B. MacIntyre, and E. Keller, 1995, “Architectural Anatomy", Presence: Teleoperators and Virtual Environments, 4(3), p. 318-325.

Fuchs, J.F.H, M. Livingston, R. Raskar, D. Colucci, K. Keller, A. State, J. Crawford, P. Rademacher, S., Drake and A. Meyer, 1998, “Augmented Reality Visualization for Laparoscopic Surgery”, Proceedings of the 1st International Conference on Medical Image Computing and Computer-Assisted Intervention (MICCAI 98), Springer-Verlag, Heidelberg, Germany, p. 934-943.

Grasset, R., and J.D. Gascuel, 2002, "MARE: Multiuser Augmented Reality Environment on table setup", SIGGRAPH, Conference Abstracts and Applications, Computer Graphics Annual Conference Series 2002, p. 213.

Habraken, N.J., and M.D. Gross, 1988, “Concept Design Games”, Design Studies, 9(3), p.181

Hillier B., J. Musgrove, and P. O'Sullivan, 1972, "Knowledge and Design", Environmental Design Research and Practice, University of California Press, Los Angeles.

Hillier, B., and A. Leaman, 1974, "How is design possible?", Journal of Architectural Research, 3(1), p. 4-11.

Hillier, B., and J. Hanson, 1984, The Social Logic of Space, Cambridge University Press, Cambridge.

Hillier, B., 1996, Space is the Machine, Cambridge University Press, Cambridge.

Hillier, B., 1998, "A Note on the intuiting of Form: Three Issues in the Theory of Design", Environment and Planning B: Planning and Design, 25, p. 37-40.

Ishii, H., B. Ullmer, 1997, “Tangible Bits: Towards Seamless Interfaces between People, Bits and Atoms", CHI'97, Atlanta, Georgia, p.234-241.

Jones, C., 1963, “A Method of Systematic Design”, Conference on Design Methods, Pergamon, Oxford.

Jones, C., 1977, "How My Thoughts about Design have Changed During the Years", Design Methods and Theories, 11(1), p.50-62. 
architectural design

Lawson, B., 1990, How Designers Think: the design process demystified, $2^{\text {nd }}$ edition, Butterworth, London.

Navab, N., B. Bascle, M. Appel, and E. Cubillo, 1999, "Scene Augmentation via the Fusion of Industrial Drawings and Uncalibrated Images with a View to Markerless Calibration", Proceedings of the 2nd International Workshop on Augmented Reality (IWAR 99), IEEE CS Press, Los Alamitos, California, p. 125-133.

Peng, C., 1994, "Designers in Teamworking”, ITRI-94-6, University of Brighton.

Penn, A., P. Treleavan, B. Hillier, L. Bull, R. Conroy, N. Dalton, L. Dekker, C. Mottram, and A. Turner, 1996, "Intelligent Architecture: Pangea, an Intelligent Workbench for Architectural Sketch Design.”, The Intelligent Systems Integration Programme ES96 Papers, SGES Publications, Oxford, p. 127-139.

Penn, A., J. Desyllas, and L. Vaughan, 1999, "The Space of Innovation: Interaction and Communication in the Work Environment", Environment and Planning B: Planning and Design, 26 (2), Pion, London, p. 193-218.

Penn, A., A. Turner, 2002, "Space Syntax based Agent Simulation", Pedestrian and Evacuation Dynamics, Springer-Verlag, p. 99-114.

Poupyrev, I., D.S. Tan, M. Billinghurst, H. Kato, H. Regenbrecht, and N. Tetsutani, 2001, "Tiles: A Mixed Reality Authoring Interface", INTERACT 2001 Conference on Human Computer Interaction, Tokyo, Japan.

Rauterberg, M., M. Fjeld, H. Krueger, M. Bichsel, U. Leonhardt, and M. Meier, 1997, "BUILD-IT: a Video-based Interaction Technique of a Planning Tool for Construction and Design", Proceedings of work With Display Units - WWDU'97, Tokyo, p. 175-176.

Regenbrecht, H.T., M.T. Wagner, and G. Baratoff, 2002, "MagicMeeting: A Collaborative Tangible Augmented Reality System”, Virtual Reality, 6, p.151-166.

Rittel, H., and M. Webber, 1973, "Dilemmas in a General Theory of Planning", Policy Sciences, 4, Elsevier, Amsterdam, p. 155-169.

Schoen, D.A., 1983, The Reflective Practitioner: How Professionals think in Action, Harper Collins, New York.

Schoen, D.A., 1987, Educating the Reflective Practitioner, Jossey-Bass, San Francisco.

Simon, H., 1969, The Sciences of the Artificial, MA: The MIT Press, Cambridge, Mass.

Simon, H., 1973, "The Structure of ill-structured Problems”, Artificial Intelligence, 4, p.181202.

Stiny, G., and L., March, 1981,“Design Machines”, Environment and Planning B: Planning and Design, 8, Pion, London, p. 213-238.

Turner, A., and A. Penn, 2002, "Encoding Natural Movement as an agent-based System: An Investigation into Human Pedestrian Behaviour in the Built Environment", Environment and Plan B: Planning and Design, 29 (4), p. 473-490.

Underkoffler, J. and H., Ishii, 1999, “ Urp: A Luminous-Tangible Workbench for Urban Planning and Design.", Proceedings of Conference on Human Factors in Computing Systems (CHI99), ACM Press, p. 386-393.

Venturi, R., 1966, Complexity and Contradiction in Architecture, The Museum of Modern Art, New York.

Wanstall, B., 1989, "HUD on the Head for Combat Pilots", Interavia, 44, p. 334-338.

Webster, A., S. Feiner, B. MacIntyre, W. Massie, and T. Krueger, 1996, "Augmented Reality in Architectural Construction, Inspection and Renovation", Proceedings of the ASCE $3^{\text {rd }}$ Congress on Computing in Civil Engineering, Anaheim, CA, p. 913-919.

Weghorst, S., 1997, "Augmented Reality and Parkinson's Disease", Communications of the $A C M, 40(8)$, p. 47-48. 